\title{
DESLOCAMENTO PARA A ESQUERDA DE ARGUMENTO INTERNO NAS LÍNGUAS GUAIKURÚ
}

\section{INTERNAL ARGUMENT LEFT DISLOCATION IN GUAYKURÚAN LANGUAGES $^{1}$}

Filomena Sandalo, Universidade Estadual de Campinas

Campinas, São Paulo, Brasil

\begin{abstract}
RESUMO: Várias línguas indígenas da América do Sul contam com o deslocamento para a esquerda de um argumento interno. Este deslocamento ocorre com argumentos de primeira ou segunda pessoa ou quando há saliência focal do objeto. Abordo o fenômeno nas línguas Guaikurú, faladas ente o Brasil, Argentina e Paraguai, e defendo que o argumento deslocado é movido para um especificador de TP.
\end{abstract}

PALAVRAS-CHAVE: Assimetrias de pessoa; Deslocamento sintático; Línguas indígenas da América do Sul

\begin{abstract}
Several South American native languages show left dislocation of an internal argument. The dislocation occurs always when the internal argument is first or second person and when there is focal prominence of the object. I approach the phenomenon in the Guaikurúan languages spoken in the boundary between Brazil, Argentina, and Paraguay. I argue that the internal argument is moved to the TP domain, along with the external argument.
\end{abstract}

KEYWORDS: Person asymmetries; Syntactic dislocation; Indigenous languages of South America

\footnotetext{
${ }^{1}$ Este trabalho foi financiado pela FAPESP, projeto 2012/17869-7, e pelo CNPq, projeto 303062/2015-Os dados foram coletados, em sua maioria, em trabalho de campo no Mato Grosso do Sul, Brasil. Agradeço principalmente Hilário e Sandra pela colaboração com os dados.
} 


\section{INTRODUÇÃO}

$\mathrm{Na}$ literatura de línguas indígenas da América do Norte, há um fenômeno conhecido como voz inversa (cf. línguas as Algonquianas, OXFORD, 2017). Estas línguas distinguem a concordância de sujeito e objeto não apenas por marcas de concordância diferentes, mas por uma hierarquia de pessoa. Para distinguir sujeito de objeto, um morfema chamado de inverso marca o verbo e o verbo passa a concordar com o objeto. A voz inversa ocorre também em línguas nativas da América do Sul, destaco aqui as línguas Guaikurú (SANDALO, 2009; VAN GYSEL, 2017) e Tupi-Guarani (PAYNE, 1994; FREITAS, 2011; ZUBIZARRETA \& PANCHEVA, 2017). Este trabalho tem como foco apenas as primeiras.

A voz inversa sintaticamente é o deslocamento de um argumento interno para a esquerda sem que haja intransitivização, como ocorre em uma passiva, por exemplo. Embora as línguas estudadas sejam radicalmente pro-drop, os argumentos internos e externos podem estar presentes e não há nenhum tipo de evidência de intransitivização.

O exemplo abaixo (de SANDALO, 2009) é da língua Kadiwéu falada no Mato Grosso do Sul. No exemplo (1), na voz direta, o verbo concorda com o sujeito, em (2) o verbo é marcado pelo morfema inverso, rotulado por relacional (REL) na literatura da linguística indígena brasileira (RODRIGUES, 1953), e o verbo concorda com o objeto.
a. Goti yema:n ekode Goti3SUBJ-amaekode 'Goti ama Ekode'
b. Goti aqa:mi Gad:ema:ni
Goti 2 PRO 2OBJ-inverso/REL-ama-pl
'Goti ama você'

Rodrigues (1953), trabalhando com fatos similares de línguas TupiGuarani, identificou o morfema inverso como 'morfema relacional de contiguidade' porque, para o autor, este morfema indicaria que o argumento de um dado núcleo lexical antecederia este núcleo. De fato, a voz inversa nas línguas brasileiras afeta concordância e ordem de constituinte como visto nos exemplos do Kadiwéu acima: os argumentos internos na voz inversa são pré-verbais, enquanto são pós-verbais na voz direta. Ou seja, o morfema relacional é presente nos mesmos casos em que o argumento interno é fronteado e podemos observar o fenômeno de contiguidade apontado por Rodrigues. ${ }^{2}$

Em kadiwéu, o fenômeno ocorre quando um argumento interno é de primeira ou segunda pessoa, como em (1), ou quando saliência focal de

\footnotetext{
${ }^{2}$ A concordância do kadiwéu é um pouco mais complexa que a reportada aqui. Para uma discussão detalhada, veja Nevins \& Sandalo (2011). E para uma discussão mais detalhada sobre o trabalho de Rodrigues, veja Sandalo (2014).
} 
objeto, fenômeno chamado de obviação (cf. SENNA, 2017), e que será discutido na seção 4. Storto (1999) discute deslocamento por foco de objeto também em karitiana, uma língua Tupi.

Embora nem todas as línguas Guaikurú apresentem o morfema inverso e concordância com o argumento fronteado, a situação de deslocamento para a esquerda de um argumento interno ocorre em todas elas.

Van Gysel (2017) discute ordem sintática em todas as línguas Guaikurú. O autor toma como base o trabalho de Carpio (2007) para a língua toba, falada na Argentina e Paraguai, e afirma que um argumento interno pronominal de primeira ou segunda pessoa precede o verbo, enquanto um argumento de terceira pessoa é pós-verbal:

a. ayim imaqtak namajipi

1PRO 3SUBJ-desprezar DEM

'Eles me desprezam'

$$
\begin{aligned}
& \text { b. sachegenaqtak na mañik lapat } \\
& \text { 1SUBJ-cortar DEM ema 3POSS-carne }
\end{aligned}
$$

'Nós estamos cortando a carne da ema'

O pilagá falado na Argentina, segundo o autor, tem um comportamento bastante similar ao kadiwéu em relação à ordem sintática, embora também não apresente o morfema inverso como o kadiwéu. Nesta língua, argumentos internos de terceira pessoa seguem o verbo e argumentos de primeira ou segunda pessoa o precedem. Os dados são de Vidal 2001.

$$
\begin{aligned}
& \text { a. amii' siayavanalo } \\
& 2 \text { PRO 1SUBJ-chamar-2plOBJ } \\
& \text { 'Eu chamo você' } \\
& \text { b. se'et doho } \\
& \text { 1SUBJ-consertar DEM } \\
& \text { 'Eu o conserto' }
\end{aligned}
$$

Finalmente, o Mocovi, também falado na Argentina, tem o morfema inverso e apresenta um fato bastante interessante para a discussão. A língua apresenta a ordem SVO (sujeito-verbo-objeto direto), mesmo quando há argumentos interno de primeira ou segunda pessoa. Mas o morfema inverso não ocorre nesta ordem sintática (exemplo 5). Se o verbo for marcado pelo morfema inverso r-, um argumento de primeira ou segunda pessoa não pode mais ser pós-verbal (exemplo 4). Os dados são de Grondona (1998) e discutidos por Van Gysel. A construção inversa parece ser opcional nesta língua. 
a. Фelisa iretagñi

Felissa 1OBJ-INV-pentear-DIR

Ela me penteia

(6)

b. Фelisa ietagñi yim

Felissa 3OBJ-pentear-DIR 1PRO

'Felissa me penteia'.

Nas seções que seguem tomo a língua kadiwéu, com base em trabalho de campo, para testes sintáticos. Tentarei mostrar evidência para acreditar que o argumento interno fronteado é deslocado para um segundo especificador do sintagma de tempo (TP).

Nesta visão, o morfema inverso é uma marca de concordância com um argumento que tenha cruzado a borda do sintagma de $v$ (vP) (cf. SANDALO, 2015). Oxford (2016) adota análise bastante similar para as línguas Algonquinas, e Zubizarreta \& Pancheva (2017) para o Guarani.

$\mathrm{O}$ interesse em entender tais construções pode ter implicações para o entendimento do português brasileiro. Como notado em Avelar e Galves (2011), o português brasileiro admite fronteamento e concordância com o argumento fronteado não comuns entre línguas românicas.

Além disso, como já vimos nesta seção, os argumentos verbais de várias línguas brasileiras de mais de uma família linguística atuam dentro de uma assimetria que contrapõe primeira e segunda pessoas vs. terceiras pessoas. É interessante salientar que Galves (2018) discute assimetrias entre argumentos de terceira pessoa e de primeira e segunda pessoa no português do Brasil que não ocorrem em português europeu.

Pode ser possível que os comportamentos mencionados do português brasileiro tenham sido favorecidos através do contato com línguas que contem com voz inversa e assimetrias de pessoa.

\section{O POSICIONAMENTO DOS ARGUMENTOS EM RELAÇÃO AO MORFEMA DE TEMPO}

As línguas Guaikurú não apresentam flexão de tempo no verbo. Apresentam palavras funcionais marcadoras de tempo/aspecto. Abaixo apresento o marcador jaG 'proximativo' do kadiwéu. Tal marcador significa que o evento aconteceu/acontecerá na linha do tempo próximo ao tempo da fala. Se contrapõe ao marcador jonaGa 'distal', significando que o evento ocorreu/ocorrerá distante do momento da fala. Em trabalhos anteriores, Sandalo (cf. SANDALO; 2009, 2015) considerou tais marcadores como advérbios, mas, em trabalho mais recente, apresenta evidência de que são palavras funcionais que dão ancoragem à sentença, isso é ocorrem em T na estrutura arbórea (SANDALO, 2018). Nestas línguas o verbo não se desloca para T. Os dados são de Sandalo (2015): 
(7) a. exabigo ja $(G)$ yema: ekode

exabigo $\mathrm{T}$ amar ekode

(8) a. exabigo aqa:mi ja(G) Gadema:ni

exabigo 2PRO $\mathrm{T}$ 2OBJ-INV-amar

b. *exabigo jaG aqa:mi Gadema:ni

exabigo T 2PRO 2OBJ-INV-amar

"Exabigo já amou você"

Note que em (6) um argumento interno de terceira pessoa é pósverbal. Já um argumento de segunda pessoa, como em (7), deve ocorrer em posição pré-verbal e pré-aspectual. O fato de o argumento interno ocorrer antecedendo o marcador de aspecto indica que houve deslocamento para uma posição alta em TP (POLLOCK, 1989).

\section{NEGAÇÃO}

A negação também ocorre à direita do argumento interno de primeira ou segunda pessoa:

(9) e: qa:mi a(G) Gadema:ni

1PRO 2PRO NEG 2OBJ-INV-amar

'Eu não te amo'

Na negação também o argumento de terceira pessoa é pós-verbal.

Há um caso em que um argumento interno de terceira pessoa pode ser pré-verbal: foco contrastivo. Mas neste caso, a negação ocorre à esquerda da terceira pessoa como mostram os dados abaixo nos dados de Sandalo(2015):

(10) exabigo aG ekode yema:

exabigo NEG ekode 3SUBJ-amar

'É Ekode (não outra) que Exabigo ama'

Note ainda que o morfema inverso ou relacional não ocorre no caso de fronteamento por foco contrastivo e a concordância permanece sendo com o sujeito.

Os dados indicam que o argumento de terceira pessoa em foco contrastivo está em um especificar de vP, enquanto um argumento interno de primeira ou segunda pessoa estão em TP. E, de fato, não há distribuição complementar entre um argumento de terceira pessoa em foco contrastivo e um argumento interno de primeira ou segunda pessoa (SANDALO, 2015):

(11) e: aqa:mi libole jolataGadomi

1PRO 2PRO 3POSS-carne 1SUBL-dar-2APLL-benefactivo

'A carne (não outra coisa), estou dando para você. 


\section{SALIÊNCIA FOCAL DO OBJETO OU OBVIAÇÃO}

Quando os argumentos de uma sentença transitiva são todos de terceira pessoa, o argumento normalmente salientado é o externo. Quando o argumento interno é salientado põe questões discursivas e pragmáticas, em Kadiwéu, usa-se a voz inversa. Neste caso, como nos outros casos de voz inversa (i.e., com argumentos internos de primeira e segunda pessoa), o verbo concorda com o argumento interno e o morfema inverso é marcado no verbo. Este fenômeno tem sido chamado de obviação na literatura das línguas algonquinas (cf. OXFORD, 2017).

Senna (2017) faz um estudo sobre obviação em kadiwéu e apresenta, como exemplo do fenômeno, duas ocorrências do verbo -idele 'lutar' na mesma narrativa:

(11) noGononotalo ikowa lidelaGawadi ikowa Exabigo naGa didele

$\mathrm{C}+\mathrm{T}+$ pl-3-encontrar-3APLL CL 3POSS-inimigo-pl CL Exabigo C+NEG 3OBJ-INV-lutar

'Quando os inimigos encontraram com Exabigo, $<\mathrm{e}>$ combateu $<\mathrm{e}>$.'

(12) ionigi ika mele ika Gabaqeni aGika ika daGa inoqe me idele

1POSS-filho CL COMP-bom CL 2POSS-trabalho NEG-CL CL NEG 3quebrar COMP 3-lutar

'Meu filho cujo trabalho é bom, não existe aquele que ele (Exabigo) não quebre ao $<\mathrm{e}>$ combater.'

Os exemplos (11) e (12) são provenientes de uma narrativa épica kadiwéu que faz parte do corpus da língua elaborado pela autora do presente texto, e que pode ser livremente acessado em http://www.tycho.iel.unicamp.br/tbf/index. Senna mostra que o exemplo (11) foi retirado de um trecho logo após Exabigo anunciar a sua esposa que seus inimigos estavam vindo. A partir desse momento, os inimigos de Exabigo passam a ser o argumento mais proeminente da narrativa. Logo, temos, no exemplo (11), na segunda sentença, um sujeito $3 \mathrm{OBV}$ e um objeto 3PROX, então a voz inversa é acionada. Em contraste, continua Senna, o exemplo (12) foi retirado de um trecho no qual o pai de Exabigo vai procurar o filho na mata. Nesse trecho, Exabigo é o assunto central, e portanto, Exabigo é o sujeito 3PROX e os inimigos de Exabigo são objeto $3 \mathrm{OBV}$ da oração. Então, a sentença fica na voz direta.

Embora os argumentos estejam frequentemente omitidos em kadiwéu (pro-drop), um argumento de terceira pessoa pode ser inicial quando o objeto for saliente.

Van Gysel (2017 nota situação semelhante no pilagá. Ele afirma que um objeto de terceira pessoa pode preceder o verbo somente em caso de o argumento externo ser indefinido e não focalizado: 
(13) hadi' yawo qoyalat

DEM mulher IMP-matar

'Alguém matou esta mulher'

Como em pilagá, em kadiwéu, apenas um argumento interno de terceira pessoa salientado pode ser pré-verbal. Diferentemente do pilagá, entretanto, o argumento externo não necessita ser indefinido.

Mas ao contrário da primeira e segunda pessoa, em ambas as línguas, o argumento de terceira pessoa não ocorre obrigatoriamente na posição pré-verbal. Embora o argumento de terceira pessoa salientado não ocorra obrigatoriamente em posição pré- verbal, os argumentos de terceira pessoas trazem forte evidência para a hipótese de movimento para especificadores múltiplos de TP porque a hipótese de que os argumentos externos e internos ocupam posições de especificadores múltiplos de $\mathrm{T}$ na voz inversa prevê que ambos os argumentos sejam igualmente visíveis para ligação. E isto é exatamente o que ocorre. Enquanto uma oração como em (14) o argumento elidido da segunda sentença é o mesmo que o sujeito da primeira, em (15), na voz inversa, o argumento elidido é o objeto da primeira sentença:

(14) ionigi ika mele ika Gabaqeni aGika ika daGa inoqe me idele

1POSS-filho CL COMP-bom CL 2POSS-trabalho NEG-CL CL NEG 3quebrar COMP 3-lutar

'Meu filho i cujo trabalho é bom, não existe aquele que $<\mathrm{e}>\mathrm{i}$ não quebre ao lutar.'

(15) noGa ikotalo ika lidelaGawa ikowa Exabigo naGa didele

$\mathrm{C}+\mathrm{T}$ 3-encontrar-APP CL 3POSS-inimigo CL Exabigo C+T 3OBJ-INV-lutar

'Quando o inimigoj encontrou Exabigoi, $<\mathrm{e}>\mathrm{i}$ combateu $<\mathrm{ej}>$.'

\section{EVIDÊNCIA INDEPENDENTE PARA O PROCESSO: ANTIPASSIVA}

Kadiwéu conta também com voz antipassiva. Nesta sintaxe, o argumento interno é demovido para uma posição externa licenciada para fora do domínio de T. A representação de uma antipassiva em Kadiwéu segundo Nevins \& Sandalo (2011) é a seguinte:

(16) Antipassiva em Kadiwéu 
Figura 1: Antipassiva em Kadiwéu

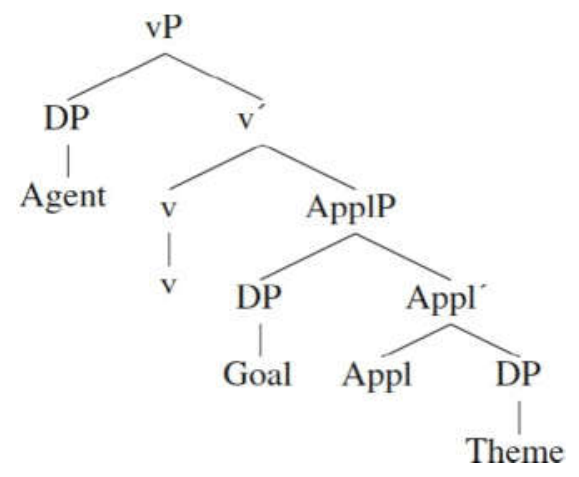

Fonte:Nevins \& Sandalo, 2011

Nesta situação, não há morfema inverso e nem fronteamento de argumento interno: o verbo é sempre marcado por um morfema de sujeito e a ordem é SVO. A antipassiva altera o significado do verbo no sentido de o objeto ser pouco afetado pela ação do verbo. Os kadiwéu traduzem as estruturas acrescentando um adjunto em português, à distância. O objeto é marcado na antipassiva por um aplicativo (APL):

(17) e: inema:ta Maria

1PRO 1INTRAN-ANT-amar-3APL

'Eu amo Maria à distância'

(18) e: inemataGawa

1PRO 1INTRAN-ANT-amar-2APLL

'Eu amo você à distância'

Tal comportamento é previsto, dado que agora os argumentos interno e externo não compartilham o domínio de $\mathrm{T}$, pois o processo de antipassivização demove o argumento interno. Note que o verbo passa a ser marcado por concordância intransitiva. $\mathrm{O}$ argumento interno ocupa a posição do especificador de APL conforme a hipótese de Nevins \& Sandalo.

\section{CONSIDERAÇÕES FINAIS: PRÓXIMOS PASSOS}

Embora o deslocamento do argumento interno exista em todas as línguas Guaikurú, a concordância do verbo transitivo é distinta em cada língua. Kadiwéu conta com distribuição complementar entre concordância de sujeito e de objeto, como vimos. O verbo concorda com o sujeito na voz direta e com o objeto na de voz inversa, embora este não seja o caso de ambos os argumentos forem de primeira e de segunda pessoa (ver NEVINS \& SANDALO, 2011 para discussão). Mas pilagá conta com concordância simultânea de sujeito e objeto. Toba concorda sempre com o sujeito. Mocovi é similar ao kadiwéu, exceto no fato de que quando os 
argumentos são de primeira e segunda, ambos são marcados, mas o de segunda será sempre um sufixo (enquanto o outro argumento é prefixal como nas outras línguas Guaikurú). É irrelevante se a segunda pessoa é o argumento interno ou externo, a segunda pessoa aparecerá com um sufixo ao verbo (Carrió, comunicação pessoal).

Nevins \& Sandalo (2011) consideram a concordância múltipla pode gerar um excesso de marcação no mesmo nó T. E a fonologia (PF) tem recursos de reajustes destas situações intoleráveis, como é bem conhecido pelos trabalhos de Ito \& Mester (2003) em restrições de co-ocorrência de traços. Sandalo (2018) desenvolve esta análise para todas as línguas Guaikurú. No caso das línguas Guaykurú, e provavelmente

Tupi-Guarani (cf. FREITAS, 2012; ZUBIZARRETA \& PANCHEVA, 2017), o argumento interno e externo estão no mesmo domínio. $^{3}$ Esta situação gera concordância múltipla de T. No caso de argumentos de primeira e segunda pessoa, gera-se uma configuração de traços de primeira pessoa inclusiva, situação bastante marcada em termos de traços. As línguas Guaykurú não aceita tal configuração e regras de PF são acionadas para reparar a configuração banida. Ou seja, segundo esta análise, a concordância é fruto de regras pós-sintáticas. Nesta perspectiva, segundo a proposta de Sandalo (2018), o kadiwéu conta com empobrecimento do traço [+autor] (veja a proposta em NEVINS \& SANDALO, 2011) e o mocovi conta com fissão e abaixamento por cópia, nas linhas de EMBICK, 2001), de [+participante].

\section{REFERÊNCIAS}

AVELAR, J.; GALVES, C. Tópico e concordância em português brasileiro e português europeu. In: FIEIS, A. \& COTINHO, A. (orgs.) Textos selecionados XXVI Encontro da Associação Portuguesa de Linguística. Lisboa: APL. 2011, p. 69-45. Disponível em: <www4.iel.unicamp.br/projetos/afrolatinos/avelar/artigos e capitulos/Avelar Galves 2011.pdf>

FREITAS, M. L. Hierarquia de Pessoa em Avá-Guarani: considerações a partir da Morfologia Distribuída. Campinas, SP: 2011. 182 pags. Dissertação de mestrado da Universidade Estadual de Campinas.

CARPIO, M. Sistemas de alineación en toba. Hermosillo, Sonora: 2007. Dissertação de mestrado da Universidade de Sonora, México.

\footnotetext{
${ }^{3}$ A análise sobre concordância em Guaikurú foi apresentada no encontro de Teoria da Gramática ANPOLL em Salvador e no Workshop on Person na Universidade de Manitoba, Winnipeg, Canadá e uma análise mais complete deve aparecer em Sandalo (2018).
} 
GALVES, C. Ensaios sobre as gramáticas do português. Campinas: Editora da UNICAMP, 2001.

GRONDONA, V. A Grammar of Mocovi. Pittsburgh, Pensilvânia: 1998. Tese de doutorado da University of Pittsburgh PhD.

ITO, J.; MESTER, A. Japanese Morphophonemics: Markedness and Word..Structure. Linguistic Inquiry Monograph 41. Cambridge, MA: MIT Press, 2003.

NEVINS, A. The Representation of Third Person and its Consequences for Person-Case Effects. Natural Language and Linguistic Theory, v. 25. 2, [s.1.], 2007, p. 273-313

NEVINS, A. Multiple Agree with clitics: person complementarrity vs. omnivorous number. Natural Language and Linguistic Inquiry, n. 29, [s.1.], 2011, p. 939-971.

NEVINS, A.; SANDALO, F. Markedness and Morphotactics in Kadiwéu [+participant] agreement. Morphology, n. 21, [s.1.], 2011, p. 351-378.

OXFORD, W. Inverse marking as impoverishment. In: KAPLAN, Aaron; KAPLAN, Abby; MIRANDA, K; MCCARVEL, and RUBIN, Edward J. Proceedings of WCCFL 34, ed. Somerville, MA: Cascadilla, 2017, p. 413-422.

PAYNE, D. The Tupí-Guaraní inverse. In: HOPPER, Paul; FOX, Barbara (Eds.). Voice: Form and Function. Amsterdam/Philadelphia: John Benjamins, 1994, p. $313-340$.

POLLOCK, J-Y. Verb Movement, Universal Grammar, and the Structure of VP. Linguistic Inquiry, Vol. 20, No. 3, [s.1.], 1989, p. 365-424.

RODRIGUES, A. Morfologia do verbo Tupi. Detalhes. Letras, Porto Alegre n. 1, 1953, p. 121-152.

RODRIGUES, A. You and I = neither you nor I: the personal system of Tupinambá. In: PAYNE, D. (ed.) Amazonian Linguistics studies in lowland South American languages. Austin: University of Texas Press. 1990, p. 393-405.

SANDALO, F. Person Hierarchy and Inverse Voice in Kadiwéu. Liames. Campinas n. 9:1, 2009, p. 27-40

SANDALO, F. Neither you nor I: the relational morpheme in person hierarchy and differential object marking in Kadiwéu. DELTA 30, São Paulo 2014, p. 645658 . 
SANDALO, F. The relational morpheme of Brazilian languages as impoverished agreement markers. In: . Working Papers in Linguistics. Univerdade de British Columbia. 2015. British Columbia, Canada, 2015, p. 92-88.

SANDALO, F. Impoverishment and fission in person asymmetries in Guaykurúan languages. Texto apresentado no congresso Manitoba Workshop on Person. Universdade de Manitoba, Canada, November 2017.

SENA, T. A. Obviação em Kadiwéu. Campinas, SP: 2017. Dissertação de Mestrado em Linguística, Universidade Estadual de Campinas.

STORTO, L. Aspects of a Karitiana Grammar. Tese de doutorado da Instituto de tecnologia de Massacusetts (MIT) 1999. 218 pags.

VAN GYSEL, J. Dissecting Alignment in South America: Hierarchical CrossReferencing Systems in the Guaykuruan Languages. Ghent, Belgica: 2016. 132 pags Tese de Mestrado, University of Gent.

VIDAL, A. Pilagá Grammar. Eugene, Oregon: 2001. 391 p. Tese de doutorado da Universidade de Oregon.

ZUBIZARRETA, M.L.; PANCHEVA, R. A formal characterization of personbased alignment: The Case of Paraguayan Guaraní. Natural Language and Linguistic Theory. [s.1.], 2017.

Filomena Sandalo FSandalo@gmail.com

Recebido em: 13 jan. 2018

Aceito em: 21 abr. 2018

Publicado em: 1 out. 2018 